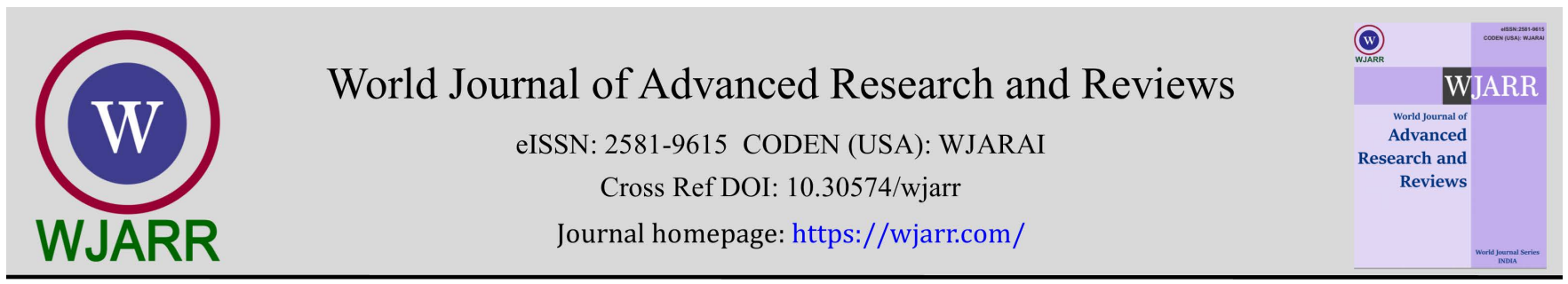

(CASE STUdY)

Check for updates

\title{
Diagnosis and treatment of gallstone intestinal obstruction in an adult patient
}

\author{
Fatin R. Polat *, Ilhan Bali, Yasin Duran and Suat Benek \\ Namık Kemal University Medical Faculty, Department of Surgery, Tekirdag, TURKEY.
}

World Journal of Advanced Research and Reviews, 2021, 09(02), 090-094

Publication history: Received on 28 January 2021; revised on 06 Februay 2021; accepted on 08 Februay 2021

Article DOI: https://doi.org/10.30574/wjarr.2021.9.2.0044

\begin{abstract}
Background: Gallstone ileus, which is called Type Vb Mirizzi Syndrome, is a rare case of mechanical intestinal obstruction observed in older patients with history of cholelithiasis or cholecystitis. Diagnostic Imaging plays an important role in the management of patients with suspected gallstone ileus. X-Ray and Abdominal Computed Tomography (CT) are the preferred modality.
\end{abstract}

Case presentation: The patient was diagnosed with gallstone ileus at the age of 45 . The case had 10 years history of biliary colic disease. The patient who is suffered from intestinal obstruction. CT demonstrated pneumobilia involving the gallbladder, a 5, $5 \mathrm{~cm}$ calcified stone in the ileum and small bowel dilatation. He underwent enterolithotomy and a huge stone was removed. When gallbladder area was checked, the gallbladder was highly adherent (to colon and stomach) and was inflamed. We suspected malignancy so multipl biopsy was taken. Two-stage treatment model was planned. After the surgery, any emerging complications were closely monitored.

Conclusions: Abdominal CT are the preferred modality for diagnosis. The main treatment for gallstone intestinal obstruction is surgery. First step enterotomy, later intented for the gallbladder. There are two type of surgical approach for gallbladder: one-stage treatment or two-stage treatment according the inflammation of gallbladder

Keywords: Gallstone; Bile ileus; Cholecystoduodenal fistula; Intestinal obstruction

\section{Introduction}

Gallstone ileus is an uncommon complication of cholelithiasis with a high morbidity and mortality rate1. It is called Mirizzi Syndrome Type Vb. Its diagnosis is difficult2. The diagnostic tests of choice are X-Ray radiograph and abdominal CT scan3.

The main treatment for gallstone intestinal obstruction is surgery. The one-stage procedure should be reserved for stabilized patients, but in cases with associated comorbidities,only enterolithotomy can represent the best option1. We report a rare case of small bowel gallstone obstruction in an 44-year-old adult patient with cholecystoduodenal fistula.

\section{Case presentation}

A 45-year-old male patient who is suffered from intestinal obstruction consult the emergency department of surgery. Vital signs were stable, and physical examination demonstrated mild tenderness in the right upper and lower quadrant. He has had multiple attacks of cholecystitis in his past.

\footnotetext{
${ }^{*}$ Corresponding author: Fatin Rüștü POLAT, MD

Associate Proffesor of Surgery.Department of Surgery Division of General SurgeryNamık Kemal University School of Medicine. Tekirdag, TURKEY.

Copyright (C) 2021 Author(s) retain the copyright of this article. This article is published under the terms of the Creative Commons Attribution Liscense 4.0.
} 
The plain X-ray film showed pneumobilia and small bowel dilatation (Figure 1). CT demonstrated pneumobilia involving the gallbladder, a 5, $5 \mathrm{~cm}$ calcified stone in the ileum and small bowel dilatation (Figure 2, 3). Pertinent positive laboratory values included elevated CRP of $38 \mathrm{mg} / \mathrm{dL}$; pertinent negative laboratory values were normal bilirubin, and white blood cell count. After a detailed explanation of possible risks including bowel resection and others, the patient signed an informed consent for operative treatment.

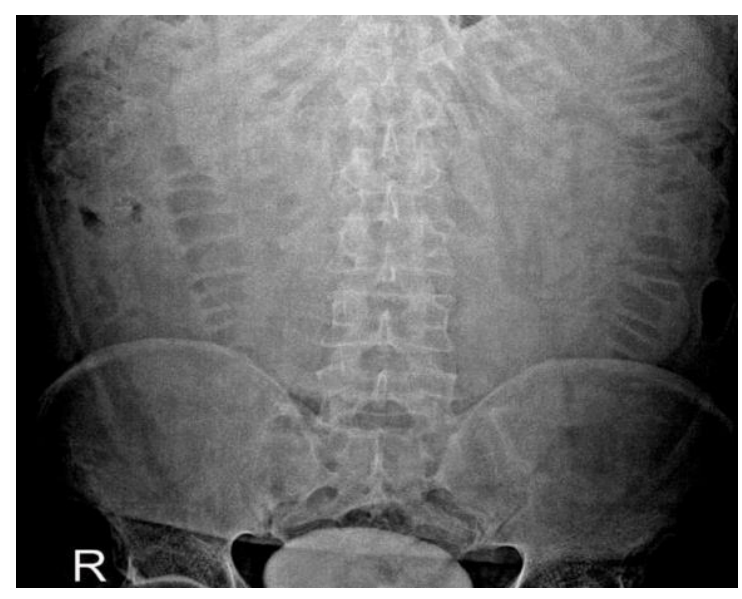

Figure 1 Plain X-ray radiograph shows air in the lumen of small intestine

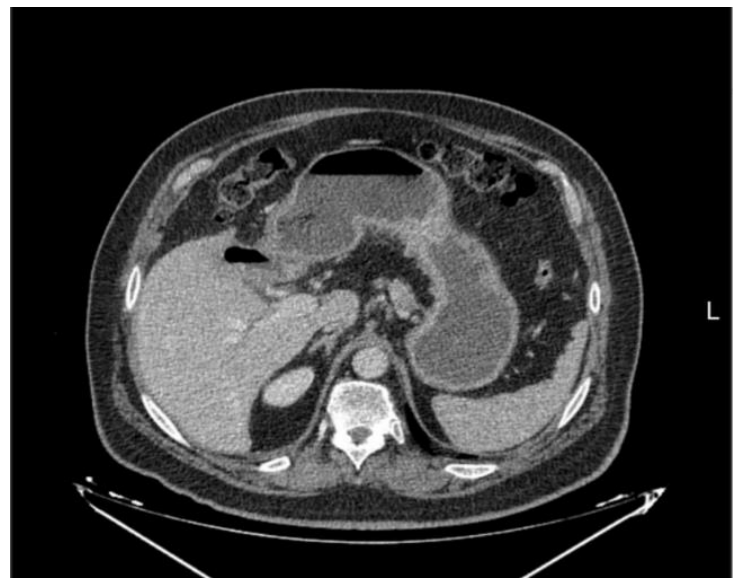

Figure 2 CT scan of the upper abdomen shows air-fluid level in the lumen of gallbladder.

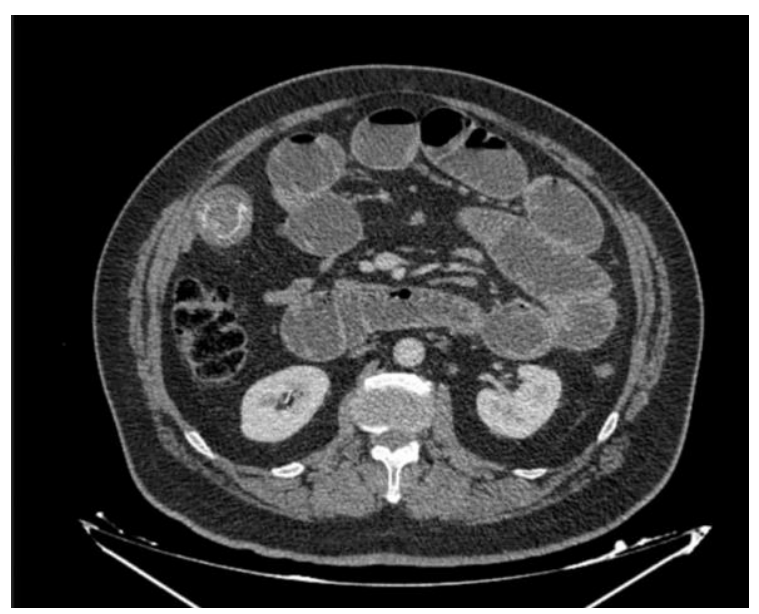

Figure 3 CT scan of the upper abdomen shows stone in the lumina of small intestine. 
The patient underwent conventional surgery under general anesthesia. A Foley catheter and a nasogastric tube were placed,During the operation, we looked for stone at the intestine. The patient underwent enterolithotomy with a longitudinal antimesenteric incision of the ileum and and a 5, $5 \mathrm{~cm}$ stone $50 \mathrm{~cm}$ from the ileocecal valve was removed (Figure 4,5,6). After that; when gallbladder area was checked, the gallbladder was highly adherent (to colon and stomach) and was inflamed. We suspected malignancy so multipl biopsy was taken. Two-stage treatment model was planned. The patient was discharged 5' th postoperative day. Our patient was discharged 6 days after surgery with a plan for future cholecystectomy and fistulectomy.

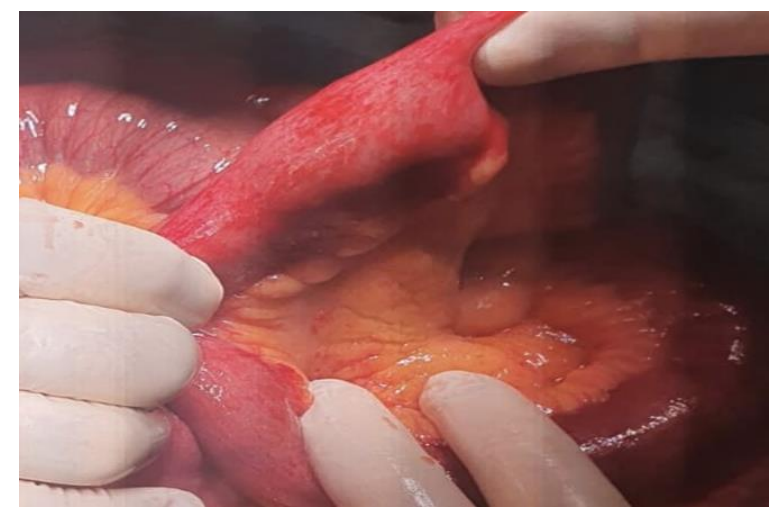

Figure 4 A solitary stone in the ileum

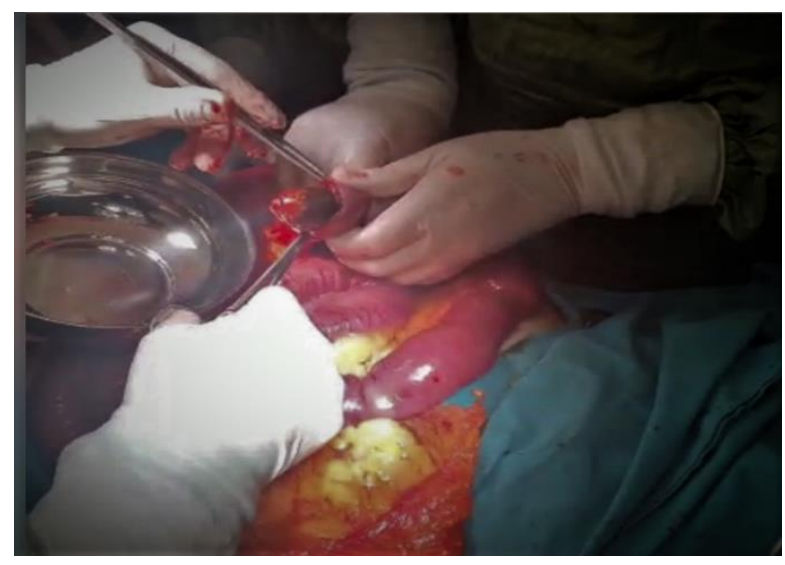

Figure 5 The patient underwent enterolithotomy with longitudinal antimesenteric incision of the duodenum and extraction of a large stone.

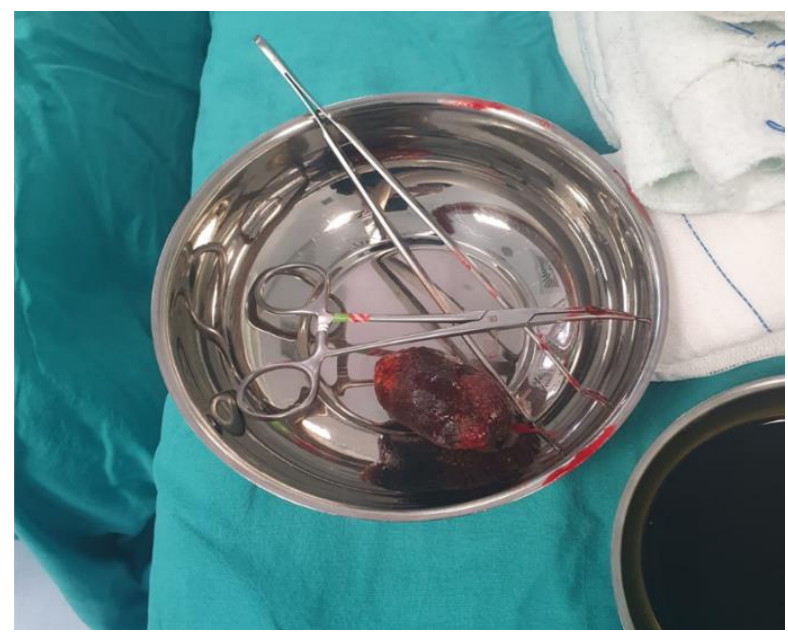

Figure 6 A solitary gallstone, measuring $5.5 \mathrm{~cm}$, was removed. 


\section{Discussion}

Symptomatic gallstones (biliary colic) and porcelain gallbladder are called the gallstone disease. The gallstone disease is an important public health problem. If gallstone disease is not treated on time, it can cause very dangerous complications with high mortality and morbidity. One of these complications is gallstone ileus.

Mirizzi syndrome is a rare complication of symptomatic cholecystitis and chronic cholelithiasis secondary to obliteration of the gallbladder infundibulum (Hartmann's bag) or the cystic duct, caused by the impact of one or more calculations5. Several types of Mirizzi syndrome were described: Type Vb refers to a cholecystoenteric fistula complicated by gallstone ileus. Our case was Type Vb Mirizzi syndrome.

The incidence of gallstone ileus is low and more common in female elderly4, whereas our case was adult. Gallstone ileus is a rare complication of cholelithiasis which occurs in less than $1 \%$ of patients and is the cause of $1-4 \%$ of cases of small bowel obstruction3. The pathogenesis involves the formation of a bilioenteric fistula.

Plain X-ray radiograph and Abdominal CT are the preferred modality for diagnosis. Abdominal radiography is rarely the primary diagnostic tool for gallstone ileus. However, at the CT in approximately $30 \%-35 \%$ of gallstone ileus patients, the diagnosis may be suggested on the basis of the clinical presentation and the Rigler triad: bowel obstruction, pneumobilia, and an ectopic gallstone5. Identification of the Rigler triad at CT is more common. Pneumobilia and a dilated stomach are easily identified. The obstructing stone is frequently radiolucent on plain films, thus complicating the differential diagnosis4, 6 .

The symptoms of gallstone obstruction are principally those of mechanical obstruction of the small bowel. The usual site of obstruction is the distal ileum. When gallstones are the cause of obstruction, the symptoms may be intermittent.

Surgical operation is the treatment of choice. Exploration should include a complete examination of the intestinal tract to make certain multiple stones are not overlooked, and the right upper quadrant should be palpated for the presence of an acutely inflamed gallbladder or more calculi.

Emergency surgery is required to treat bowel obstruction. The stone in the small intestine is removed by laparoscopic or open enterotomy. In patients with good general condition, cholecystoenteric fistula is also treated and cholecystectomy is performed in the same session (one-stage treatment). If the general condition of the patient is impaired (high risk patient), fistula treatment and cholecystectomy are left after 4-6 weeks (two-stage treatment). In addition, if gallbladder malignancy is considered, the surgery for the gallbladder is left after 6 weeks.

\section{Declarations}

- List of abbreviations: Abdominal Computed Tomography (CT)

- Ethics approval and consent to participate: Not applicable (retrospective study)

- Competing interests: None

- Funding: None

\section{Conclusion}

As a result a timely diagnosis with imaging is possible and important. The diagnosis may be made with Plain X-Ray radiograph and CT. The main treatment is surgery. Surgical method should be chosen according to the patient's condition and malignancy risk.

\section{Compliance with ethical standards}

\section{Acknowledgments}

None

\section{Disclosure of conflict of interest}

The authors has no conflict of interest to declare. 


\section{Statement of informed consent}

"Informed consent was obtained from all individual participants included in the study."

\section{References}

[1] Vasilescu A, Cotea E, Palaghia M, Vintilă D, Târcoveanu FE. Gallstone ileus: a rare cause of intestinal obstruction case report and literature review. Chirurgia (Bucur). Sep-Oct 2013; 108(5): 741-4.

[2] Artioli G, Muri M, Praticò FE, Marcantoni EA, Gazzani SE, Lana S, Bacchini E, Capretti G, Muri M, Torri T. Gallstone ileus: literature review. Acta Biomed. 28 Jul 2016; 87(3): 40-4.

[3] Zappia F, Petracca G, Talarico CA. Gallstone ileus. A case treated with minilaparotomy and a review of the literature. Ann Ital Chir. 29 Mar 2017; 6.

[4] He JJ, Xu KQ, Tang JH. [Intestinal obstruction caused by gallstone: report of 5 cases and review of the literature]. Zhonghua Wei Chang Wai Ke Za Zhi. Oct 2010; 13(10): 751-4.

[5] Fernanda Gutiérrez., et al. Mirizzi Syndrome. Case Report. EC Gastroenterology and Digestive System 8(1) 2021; 39-45.

[6] Brennan GB, Rosenberg RD, Arora S. Bouveret syndrome. Radiographics. Jul-Aug 2004; 24(4): 1171-5. 